\title{
Can heart rate variability become a screening tool for anesthesia-related hypotension?
}

\author{
M. F. HANEY ${ }^{1}$ and U. WIKLUND ${ }^{2}$ \\ ${ }^{1}$ Anesthesiology and Intensive Care Medicine and ${ }^{2}$ Biomedical Engineering and Informatics, University Hospital of Umeå and Umeå University, \\ Umea, Sweden
}

$\mathrm{H}$ EART rate variability (HRV) analysis as a diagnostic tool has been available for decades (1), and many have also learned about modern HRV analysis as a predictive or prognostic instrument for cardiological patients with diagnoses such as congestive heart failure or post-myocardial infarction (2). Numerous recent publications in the field of anesthesiology have presented findings concerning HRV as a potential tool for predicting acute circulatory disturbances, and particularly hypotension (3-11). As all in the field of anesthesiology and critical care medicine traditionally have in mind a central clinical theme to 'avoid hypotension and hypoxia' in all perioperative patients, it is relevant for the specialty to carefully study and try to establish validity for any diagnostic instruments which show promise in predicting circulatory disturbances, including hypotension.

In this (12) and the previous issue (13) of Acta Anaesthesiologica Scandinavica, two studies are presented where HRV analysis is examined as a possible screening tool for anesthesia-related hypotension $(12,13)$. Both studies present findings where a preoperative HRV finding was associated with more hypotension during spinal anesthesia. The study by Hanss et al. (12) is a continuation in a series of studies in obstetrical patients, where they have systematically tested HRV parameters in relation to different parts of the peri-operative procedure in a highly selected patient group which is at high risk of hypotension. The study by Fujuwara et al. (13) includes use of a HRV analysis method which has different characteristics from the more commonly used fast Fourier transformation (FFT) method. Both studies provide information and experience which contribute to the process of evaluation and validation of HRV as a clinical tool for assessment of perioperative patients.

The few studies published so far where HRV has been applied to predict hypotension can be regarded as preliminary studies within a new research area. Can hypotension during spinal anesthesia or other types of anesthesia be predicted from patterns in HRV? Moreover, is increased low frequency (LF) to high frequency $\mathrm{HF}$ power spectrum density ratio (LF/HF) a marker of an increased risk of hypotension, which is suggested by the findings in both studies in this issue? Is increased LF/HF caused by increased sympathetic activity, or is it mainly caused by reduced vagal outflow? Fujiwara et al. (13) also found that subjects with a low degree of randomness in their HRV seemed to be more susceptible to decreased blood pressure after spinal anesthesia. Although not specifically discussed in the paper, these subjects also had relatively low values of both LF and HF power, which was presented as absolute values. The latter finding could also indicate reduced vagal outflow in this subgroup.

HRV results in this clinical anesthesia context are difficult to interpret because of several problems, some new, and some not so new (14). First, it is not clear exactly which phenomena in the body that HRV is measuring. Second, so far there are no findings to help to understand whether HRV is more useful in predicting hypotension then other already readily available (and much simpler) parameters. Including, for example, resting heart rate before spinal anesthesia. Third, some of the methodology is difficult to examine and understand.

The first and perhaps most important issue concerns exactly which physiological or pathophysiological process HRV is assessing. Regulation of heart 


\section{F. Haney and U. Wiklund}

rate intervals and blood pressure are complex physiological process. A common theme in discussions which try to relate HRV results to blood pressure changes is that the autonomic nerve system has a form of final general expression which can be described quantitatively as the summarized balance between parasympathetic and sympathetic nerve system activity. Furthermore, one particular HRV parameter reflects this parasympathetic/sympathetic nerve system activity balance. Unfortunately, this has not been proved.

It is widely accepted that there are continuous fluctuations or oscillations in blood pressure and heart rate intervals in health, and vigorous study of these oscillation has occurred (15) which show that vagal and sympathetic cardiac autonomic modulation are reflected in different frequency regions in the HRV power spectrum. Comparing amounts of activity in these frequency regions through complicated mathematical algorithms is the basis for trying to describe a single parameter or mathematical ration of vagal and sympathetic balance as a general index of circulatory control. These different spectral components, low frequency, very low frequency, and high frequency, are the model for analysis of HRV, and this makes the model seem very neat and easy to understand. However, we must recognize that there is still much that is not appreciated about different factors, both physiological (reflexes, non-neural mechanisms, etc.) and pathophysiological (illness, medications, etc.) that affect beat-to-beat fluctuations in heart rate and occurrences in these frequency regions (15). The belief that these HRV components are 'true' and validated may have arisen from the general acceptance of large studies of the prognostic value of HRV in prediction of outcomes in selected cardiology patient groups. Quantitative assessment of autonomic outflow is still a field in its infancy, and validation of the tools of assessment is ongoing. Also note that HRV mirrors modulation, not absolute levels of autonomic activity.

It may be early to implement HRV parameters as a standard routine in clinical anesthesia patient evaluation if the mechanisms of changes in the HRV parameters themselves are not well understood. Still, HRV parameters are among the best tools available for assessment of autonomic nerve system activity and clinical application of HRV (even as an empiric measure) to examine the circulatory phenomena in peri-operative subjects clearly shows promise.

Another discussion point concerning HRV methodology has to do with forms of analysis. The disparity and variability in short-term measures of HRV have produced concern regarding methodology and forms of analysis (16). Investigators have often attempted to try to manage and simplify the analysis of their HRV results, presumably to make them easier for a reader to appreciate. Many have tried this by 'normalizing' the results - which is akin to presenting the results as relative changes in individual subjects over time, but not as absolute HRV values. Unfortunately, there is a potential for uncoupling of results from the physiological phenomena if only this type of data is interpreted. Absolute values of the raw HRV data need to be demonstrated if the readers are to be able to later interpret 'normalized' results. 'Normalizing' is often applied because there is some codependence of HRV parameters related to heart rate frequency ranges which can make results more difficult to interpret. For example, HRV normally decreases at higher heart rates.

A second issue with HRV analysis methodology concerns the mathematical means to reduce HRV phenomena to a single number. In Fujiwara et al. (13), one of the methods to do this is an alternative to FFT, which has been incorporated into a relatively new commercial product, but about which there is little published experience. The method is described as calculating entropy which is done from relatively few heart beats, at least compared with common and traditional applications of HRV. Results from FFT analysis for the same data are also included by Fujiwara et al. (13), for reference.

Analysis of entropy is a mathematically different approach from FFT, which has among other things, the superficial advantage of being very simple in its result, and apparently lesser amounts of HRV data needed for analysis compared with FFT, which is attractive in a clinical context. However, it is important to stress that analyses of short data sequences could give large fluctuations in the calculated HRV variables in most healthy subjects. On the other hand, a subject with reduced HRV will probably have low values for HRV indices irrespective of the length of the recording. Moreover, the entropy analysis also suffers from the same general weakness as described earlier, which is that it is not clear exactly which aspects of autonomic nerve system expression the entropy results are describing.

The potential or opportunity to predict perioperative hypotension is very exciting. HRV as a clinical diagnostic instrument for peri-operative patients shows promise, although more basic physiological study as well as clinical (so far, empiric) experience is needed for further validation of HRV 
before it can be recommended as a standard part of our diagnostic or monitoring armamentarium. These types of studies should also awaken enthusiasm amongst anesthesiologists for examining other readily available peri-operative parameters in the context of trying to predict hypotension.

One of our tasks as practitioners is to be clinical experts in applied physiology of the circulation. Anesthesia personnel face perhaps an unique challenge in evaluating patients not only with complex regulation of heart rate and blood pressure, not only with illnesses and medications which affect regulation and behavior of the circulation, but also concerning coming treatments (anesthetic drugs and surgical interventions) which rapidly and profoundly can change the ability of a patient's circulatory system to reflexively respond to challenges and provocations. To date, anesthesia personnel have relied largely on general impressions (intuition) or anecdotal experience to predict undesirable circulatory events, although without support of specific assessment of the autonomic nerve system. Better understanding of the resting pre-operative autonomic nerve system condition and performance capabilities, yes, let us hope for it!

\section{References}

1. Hon EH, Lee ET. Electronic evaluation of the fetal heart rate patterns preceding fetal death: further observations. Am J Obstet Gynecol 1965; 87: 814-26.

2. Task Force of the European Society of Cardiology and the North American Society of Pacing and Electrophysiology. Heart rate variability statndards of measurement, physiological interpretation, and clinical use. Eur Heart J 1996; 17: 354-81.

3. Poyhonen M, Syväoja S, Hartikainen J, Ruokonen E, Takala J. The effect of carbon dioxide, respiratory rate and tidal Volume on human heart rate variability. Acta Anaesthesiol Scand 2004; 48: 93-101.

4. Fujiwara Y, Asakura Y, Shibata Y, Nishiwaki K, Komatsu T. A marked decreases in heart rate variability associated with junctional rhythm during anesthesisia with sevoflurane and fentanyl. Acta Anaesthesiol Scand 2006; 50: 509-11.

5. Huang CJ, Kuok CH, Kuo TBJ, Hsu YW, Tsai PS. Preoperative measurement of heart rate variability predicts hypotension during general anesthesia. Acta Anaesthesiol Scand 2006; 50: 542-8.

6. Luginbuhl M, Yppärilä-Wolters H, Rufenacht M, PetersenFelix S, Korhonen I. Heart rate variability does not discriminate between different levels of haemodynamic responsiveness during surgical anesthesia. Br J Anaesth 2007; 98: 728-36.

7. Fujiwara Y, Ito H, Asakura Y, Sata Y, Nishiwake K, Komatsu T. Preoperative ultra short-term entropy predicts arterial blood pressure fluctuation during the induction of anesthesia. Anesth Analg 2007; 104: 853-6.

8. Fujiwara Y, Kurokawa S, Asakura Y, Wakao Y, Nishiwaki K, Komatsu T. Correlation between heart rate variability and hemodynamic fluctuation during induction of general anaesthesia: comparison between linear and non-linear analysis. Anaesthesia 2007; 62: 117-21.

9. Hanss R, Bein B, Francksen $\mathrm{H}$ et al. Heart rate variabilityguided prophylactic treatment of severe hypotension after subarachnoid block for elective cesarean delivery. Anesthesiology 2006; 104: 635-43.

10. Hanss $\mathrm{R}$, Bein $\mathrm{B}$, Weseloh $\mathrm{H}$ et al. Heart rate variability predicts severe hypotension after spinal anesthesia. Anesthesiology 2006; 104: 537-45.

11. Hanss $R$, Bein $B$, Ledowski $T$ et al. Heart rate variability predicts severe hypotension after spinal anesthesia for elective cesarean delivery. Anesthesiology 2005; 102: 1086-93.

12. Hanss R, Ohnesorge H, Kaufmann $M$ et al. Changes in heart rate variability may reflect sympatholysis during spinal anesthesia. Acta Anaesthesiol Scand 2007; 51: 1297-1304.

13. Fujiwara Y, Sato Y, Shibata Y, Asakura Y, Nishiwakai K, Komatsu T. Greater decreases in blood pressure after spinal anaesthesia in patients with low entropy of RR interval. Acta Anaesthesiol Scand 2007; 51: 1161-5.

14. Smiley R. Fast fourier transforms as prophecy: predicting hypotension during spinal anesthesia. Anesthesiology 2005; 102: 1079-80.

15. Parati G, Mancia G, Di Rienzo M, Castiglioni P, Taylor JA, Studinger P. Point-counterpoint: cardiovascular variability is/is not an index of autonomic control of the circulation. J Appl Physiol 2006; 101: 676-82.

16. Sandercock GRH, Bromley PD, Brodie DA. The reliability of short-term measurements of heart rate variability. Int J Cardiol 2005; 103: 238-47.

Address:

M. F. Haney

University Hospital of Umeå

Norrlans Universitetsjukhus

90185 Umea

Sweden

e-mail: michael.haney@anestesi.umu.se 